\title{
Jet-induced star formation in 3C 285 and Minkowski’s Object ${ }^{\star}$
}

\author{
Q. Salomé, P. Salomé, and F. Combes
}

\author{
LERMA, Observatoire de Paris, CNRS UMR 8112, 61 avenue de l'Observatoire, 75014 Paris, France \\ e-mail: quentin. salome@obspm.fr
}

Received 5 September 2014 / Accepted 6 November 2014

\begin{abstract}
How efficiently star formation proceeds in galaxies is still an open question. Recent studies suggest that active galactic nucleus (AGN) can regulate the gas accretion and thus slow down star formation (negative feedback). However, evidence of AGN positive feedback has also been observed in a few radio galaxies (e.g. Centaurus A, Minkowski's Object, 3C 285, and the higher redshift 4C 41.17). Here we present $\mathrm{CO}$ observations of $3 \mathrm{C} 285$ and Minkowski's Object, which are examples of jet-induced star formation. A spot (named $3 \mathrm{C} 285 / 09.6$ in the present paper) aligned with the $3 \mathrm{C} 285$ radio jet at a projected distance of $\sim 70 \mathrm{kpc}$ from the galaxy centre shows star formation that is detected in optical emission. Minkowski's Object is located along the jet of NGC 541 and also shows star formation. Knowing the distribution of molecular gas along the jets is a way to study the physical processes at play in the AGN interaction with the intergalactic medium. We observed CO lines in 3C 285, NGC 541, 3C 285/09.6, and Minkowski's Object with the IRAM $30 \mathrm{~m}$ telescope. In the central galaxies, the spectra present a double-horn profile, typical of a rotation pattern, from which we are able to estimate the molecular gas density profile of the galaxy. The molecular gas appears to be in a compact reservoir, which could be evidence of an early phase of the gas accretion after a recent merger event in $3 \mathrm{C} 285$. No kinematic signature of a molecular outflow is detected by the $30 \mathrm{~m}$ telescope. Interestingly, 3C 285/09.6 and Minkowski's Object are not detected in CO. The cold gas mass upper limits are consistent with a star formation induced by the compression of dense ambient material by the jet. The depletion time scales in 3C 285/09.6 and Minkowski's Object are of the order of and even shorter than what is found in 3C 285, NGC 541, and local spiral galaxies $\left(10^{9} \mathrm{yr}\right)$. The upper limit of the molecular gas surface density in 3C 285/09.6 at least follows a Schmidt-Kennicutt law if the emitting region is very compact, as suggested by the $\mathrm{H} \alpha$ emission, while Minkowski's Object is found to have a much higher star formation efficiency lower limit (very short depletion time). Higher sensitivity is necessary to detect CO in the star-forming spots, and higher spatial resolution is required to map the emission in these jet-induced star-forming regions.
\end{abstract}

Key words. methods: data analysis - galaxies: evolution - galaxies: interactions - galaxies: star formation - radio lines: galaxies

\section{Introduction}

The role played by active galactic nucleus (AGN) in galaxy evolution (and formation) has become a key question in the field of extragalactic astronomy in the past decade. The most often studied phenomenon is the so-called AGN feedback, which refers to a negative feedback. In other words, the energy released by the AGN (mechanical or radiative) is assumed to be transferred to the surrounding medium and as a consequence to prevent or regulate star formation, either by heating or by expelling the gas reservoir available to fuel star formation (e.g. Fabian 2012; Heckman \& Best 2014, and references therein). However, how the AGN interacts with the gas of the host galaxy in detail is still unclear. Another mechanism often referred to as AGN positive feedback is also possible. The statistical study of several hundreds of AGN by Zinn et al. (2013) showed that AGN with pronounced radio jets have a much higher star formation rate than those selected purely by X-ray. Supported by the morphological association of AGN-jets and star-forming regions (Best \& Heckman 2012; Ivison et al. 2012), it is expected that the propagation of shocks that are generated by the jets can accelerate the gas cooling and thus trigger star formation. Such radio-jet/star formation associations were observed along radio-jets of local brightest cluster galaxies (McNamara \& O'Connell 1993), and

* Based on observations carried out with the IRAM 30 m telescope. IRAM is supported by INSU/CNRS (France), MPG (Germany), and IGN (Spain). molecular gas was mapped along the radio jet of the Abell 1795 central galaxy (Salomé \& Combes 2004). In the early Universe, Emonts et al. (2014) searched for $\mathrm{CO}$ in 13 high- $z$ radio galaxies with redshifts between 1.4 and 2.8. The authors found $\mathrm{CO}$-jet alignment in several of their sources and discussed possible explanations among which were jet-induced star formation and gas cooling. Klamer et al. (2004) also discussed this interpretation for detections of molecular gas that is spatially and kinematically offset from the central galaxy (and preferentially aligned along the radio axis) in $z>3$ sources. Jet-triggered star formation processes (AGN positive feedback) were also modelled with numerical simulations. Fragile et al. (2004) used hydrodynamic simulations of radiative shock-cloud interactions to show that it is possible to very efficiently cool a large portion of gas masses along the shock propagation path. This was also discussed in detail by Gaibler et al. (2012).

However, as mentioned above, evidence of radio-jet and molecular gas interaction has been found in very few objects: (1) Centaurus A, where the jet encounters gas in the shells along its way (Schiminovich et al. 1994; Charmandaris et al. 2000); (2) Minkowski's Object (van Breugel et al. 1985); (3) 3C 285 (van Breugel \& Dey 1993); and (4) at $z=3.8$, the radio source 4C 41.17 (Bicknell et al. 2000; De Breuck et al. 2005; Papadopoulos et al. 2005). Some other systems are suspected to belong to these objects in cooling flow clusters, such as Abell 1795 (McNamara 2002; Salomé \& Combes 2004), or Perseus A (Salomé et al. 2008, 2011) for instance. To better 
Table 1. General properties of 3C 285 and 3C 285/09.6.

\begin{tabular}{lcc}
\hline \hline Source & 3C 285 & 3C 285/09.6 \\
\hline$z$ & \multicolumn{2}{c}{$0.0794^{a}$} \\
$D_{\mathrm{A}}(\mathrm{Mpc})$ & \multicolumn{2}{c}{309} \\
$D_{\mathrm{L}}(\mathrm{Mpc})$ & \multicolumn{2}{c}{360} \\
Scale $\left(\mathrm{kpc} /{ }^{\prime \prime}\right)$ & \multicolumn{2}{c}{1.5} \\
\hline $\mathrm{RA}(\mathrm{J} 2000)$ & $13^{\mathrm{h}} 21^{\mathrm{m}} 17.813$ & $13^{\mathrm{h}} 21^{\mathrm{m}} 22^{\mathrm{s}} .134$ \\
Dec $(\mathrm{J} 2000)$ & $+42: 35: 15.38$ & $+42: 35: 20.14$ \\
$v_{\mathrm{Hel}}\left(\mathrm{km} \mathrm{s}^{-1}\right)$ & $0(z=0.0794)$ & $-132 \pm 25^{a}$ \\
$m_{\mathrm{B}}\left(\mathrm{mag}^{a}\right)$ & $16.86^{b}$ & \\
$L_{\mathrm{H} \alpha}\left(\mathrm{erg} \mathrm{s}^{-1}\right)$ & $2.5 \times 10^{41 c}$ & $2.8 \times 10^{40 a}$ \\
$S F R_{\mathrm{H} \alpha}\left(M_{\odot} \mathrm{yr}^{-1}\right)$ & $1.34^{d}$ & $0.15^{d}$ \\
$L_{24 \mu \mathrm{m}}\left(\mathrm{erg} \mathrm{s}^{-1}\right)$ & $1.1 \times 10^{44}$ & $3.8 \times 10^{42}$ \\
$S F R_{24 \mu \mathrm{m}}\left(M_{\odot} \mathrm{yr}^{-1}\right)$ & $12.0^{e}$ & $0.61^{e}$ \\
$L_{\mathrm{TIR}}\left(L_{\odot}\right)$ & $1.32 \times 10^{11}$ & $<5.3 \times 10^{9}$ \\
$S F R_{\mathrm{TIR}}\left(M_{\odot} \mathrm{yr}^{-1}\right)$ & $19.7^{d}$ & $<0.79^{d}$ \\
\hline
\end{tabular}

Notes. The TIR luminosity was computed on the $3-1100 \mu \mathrm{m}$ range. The $24 \mu \mathrm{m}$ luminosity comes from the WISE archive. The $\mathrm{H} \alpha$ luminosity is extinction corrected for 3C 285/09.6, but not for 3C 285 .

References. ${ }^{(a)}$ van Breugel \& Dey (1993); ${ }^{(b)}$ Véron-Cetty \& Véron (2010); ${ }^{(c)}$ Baum \& Heckman (1989); ${ }^{(d)}$ Kennicutt \& Evans (2012); ${ }^{(e)}$ Calzetti et al. (2007).

understand the physical processes at play in the AGN interaction with the intergalactic medium and its impact on star formation, it is important to know the molecular gas distribution in these objects.

3C 285 is a double-lobed powerful FR-II radio galaxy where both lobes have a complex filamentary structure. In the eastern radio lobe, there is a radio jet with unresolved radio knots (van Breugel \& Dey 1993). A slightly resolved object is located near the eastern radio jet (3C 285/09.6; van Breugel \& Dey 1993; also identified as SDSS J132122.14+423519.8). 3C 285/09.6 is a small, kiloparsec-sized object where star formation seems to be triggered by the jet from the radio source 3C 285 (van Breugel $\&$ Dey 1993). Table 1 summarises general properties of the radio galaxy 3C 285 and 3C 285/09.6.

Minkowski's Object (MO) is a star-forming peculiar object near the double-lobed FR-I radio source NGC 541 in the galaxy cluster Abell 194 (Croft et al. 2006). In projection, MO is located in a large optical bridge that connects NGC 541 with the interacting galaxies NGC 545/547, suggesting that gas of MO may have origins in previous interactions between these galaxies. MO has recently been observed but not detected in $\mathrm{CO}(1-0)$ with the Plateau de Bureau interferometer (Nesvadba et al., in prep.). VLA observations show two HI clouds "wrapped" around the eastern jet with a total mass of $4.9 \times 10^{8} M_{\odot}$ (Croft et al. 2006). This suggests that HI may be the result of radiative cooling of warmer gas in the IGM. This highlights a major difference with Centaurus A: in Cen A, the jet probably hits an existing HI cloud (Mould et al. 2000), whereas the jet of NGC 541 may have caused warm gas to cool forming HI. General properties of NGC 541 and MO are summarised in Table 2.

$\mathrm{CO}(1-0)$ and $\mathrm{CO}(2-1)$ have been observed along the jet axis of the radio galaxies 3C 285 and NGC 541. Our main goal was to determine the star formation efficiency (SFE) in the galaxies and inside the jets and determine whether star formation is more efficient in the shocked region along the jet.

In Sect. 2, we present the data used for this study. The results derived from the observations (Sect. 3) are then discussed in Sect. 4. Throughout this paper, we assume the cold dark matter
Table 2. General properties of NGC 541 and Minkowski's Object.

\begin{tabular}{lcc}
\hline \hline Source & NGC 541 & Minkowski's Object \\
\hline$z$ & $0.0181^{a}$ & $0.0189^{a}$ \\
$D_{\mathrm{A}}(\mathrm{Mpc})$ & 75.8 & 79.1 \\
$D_{\mathrm{L}}(\mathrm{Mpc})$ & 78.6 & 82.1 \\
Scale $\left.(\mathrm{kpc})^{\prime \prime}\right)$ & 0.37 & 0.38 \\
\hline $\mathrm{RA}(\mathrm{J} 2000)$ & $01^{\mathrm{h}} 25^{\mathrm{m}} 44.3$ & $01^{\mathrm{h}} 25^{\mathrm{m}} 47.5$ \\
Dec $(\mathrm{J} 2000)$ & $-01: 22: 46.4$ & $-01: 22: 20.0$ \\
$m_{\mathrm{B}}(\mathrm{mag})$ & $13.0^{b}$ & $17.5^{b}$ \\
\hline$L_{\mathrm{H} \alpha}\left(\mathrm{erg} \mathrm{s}^{-1}\right)$ & $1.3 \times 10^{39 c}$ & $6.6 \times 10^{40 a}$ \\
$S F R_{\mathrm{H} \alpha}\left(M_{\odot} \mathrm{yr}^{-1}\right)$ & $7 \times 10^{-3 d}$ & $0.35^{d}$ \\
$L_{24 \mu \mathrm{m}}\left(\mathrm{erg} \mathrm{s}^{-1}\right)$ & $7.4 \times 10^{41}$ & $6.6 \times 10^{41}$ \\
$S F R_{24 \mu \mathrm{m}}\left(M_{\odot} \mathrm{yr}^{-1}\right)$ & $0.14^{e}$ & $0.13^{e}$ \\
$L_{\mathrm{FIR}}\left(L_{\odot}\right)$ & - & $5.2 \times 10^{8 f}$ \\
$S F R_{\mathrm{FIR}}\left(M_{\odot} \mathrm{yr}^{-1}\right)$ & - & $0.09^{g}$ \\
\hline
\end{tabular}

Notes. The $\mathrm{H} \alpha$ luminosity is not corrected for extinction.

References. ${ }^{(a)}$ Croft et al. (2006); (b) Simkin (1976); ${ }^{(c)}$ Capetti et al. (2005); ${ }^{(d)}$ Kennicutt \& Evans (2012); ${ }^{(e)}$ Calzetti et al. (2007); ${ }^{(f)}$ Engelbracht et al. (2008); ${ }^{(g)}$ Kennicutt (1998).

concordance Universe, with $H_{0}=70 \mathrm{~km} \mathrm{~s}^{-1} \mathrm{Mpc}^{-1}, \Omega_{\mathrm{m}}=0.30$ and $\Omega_{\mathrm{A}}=0.70$.

\section{Observations}

\subsection{IRAM $30 \mathrm{~m}$, Pico Veleta}

Millimetre observations of the $\mathrm{CO}(1-0)$ and $\mathrm{CO}(2-1)$ emission were made with the IRAM $30 \mathrm{~m}$ telescope in March and June 2014. At redshift $z=0.0794$ (resp. $z=0.0181$ ), these lines are observable at frequencies of $106.780 \mathrm{GHz}$ (resp. 113.220 GHz) and $213.580 \mathrm{GHz}$ (resp. $226.439 \mathrm{GHz}$ ), which leads to beams of $24^{\prime \prime}$ and $12^{\prime \prime}$ (resp. 22" and $11^{\prime \prime}$ ). The EMIR receiver ${ }^{1}$ was used simultaneously with the $4 \mathrm{MHz}$, FTS and WILMA backends (bandwidths of $4 \mathrm{GHz}, 4 \mathrm{GHz}$ and $3.7 \mathrm{GHz}$; resolution of $4 \mathrm{MHz}, 195 \mathrm{kHz}$ and $2 \mathrm{MHz}$ respectively). Owing to instrumental problems, the FTS backend could not be used. Moreover, the $4 \mathrm{MHz}$ backend was only prepared for the $\mathrm{CO}(1-0)$ line. Therefore, only the WILMA backend is used in this paper.

During observations, the pointing was monitored by observing Mars and standard continuum sources tuned to the frequency corresponding to the redshifted $\mathrm{CO}(1-0)$ emission line. Observations were obtained using wobbler switching with a rate of $\sim 0.5 \mathrm{~Hz}$. Six-minute scans were taken, and a calibration was made every three scans. Pointing was checked every few hours by observing standard continuum sources and was generally determined to be accurate to within a few arcseconds.

Three regions were observed in March 2014: the central galaxy 3C 285, 3C 285/09.6, and an intermediate position (3C 285-2) along the jet (cf. Fig. 1). Along observing nights, the system temperature remained good. It varied between 100 and $165 \mathrm{~K}$ for the $\mathrm{CO}(1-0)$ line and was in the range $240-510 \mathrm{~K}$ for $\mathrm{CO}(2-1)$. In June 2014, observations pointed at NGC 541 and Minkowski's Object (cf. Fig. 2), with system temperatures of $180-200 \mathrm{~K}$ for $\mathrm{CO}(1-0)$ emission and $210-250 \mathrm{~K}$ for the $\mathrm{CO}(2-1)$ line. To reach a better rms, our data of NGC 541 were combined with those of Ocaña Flaquer et al. (2010). Observation conditions are summarised in Table 3.

\footnotetext{
1 http://wWw . iram.es/IRAMES/mainWiki/ EmirforAstronomers
} 


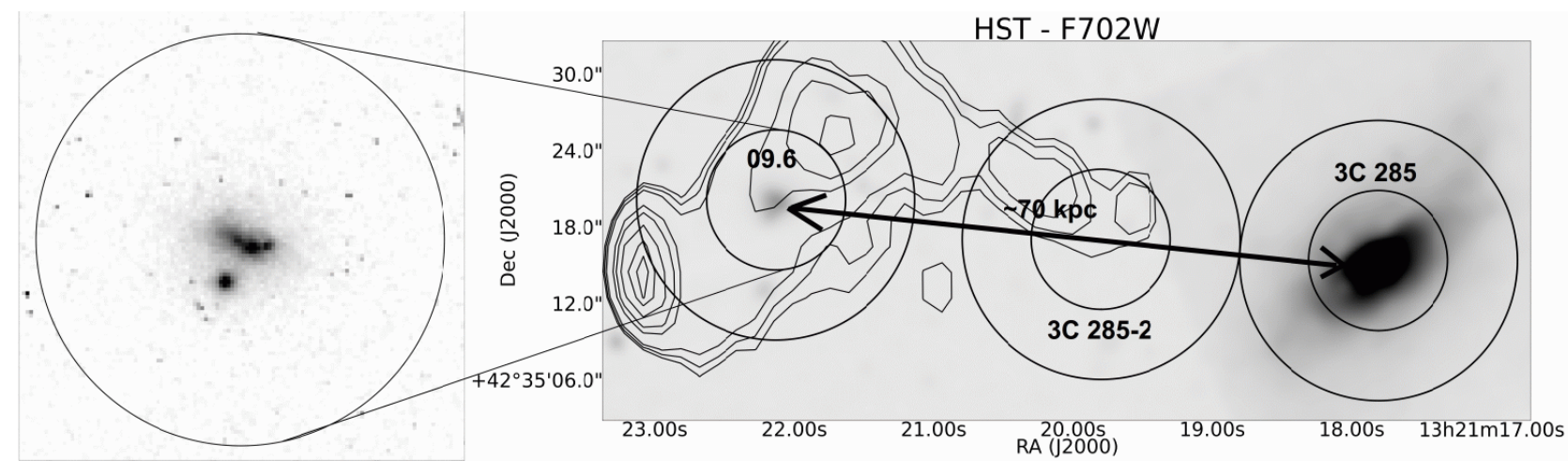

Fig. 1. Contour map of the eastern lobe of 3C 285 observed at $21 \mathrm{~cm}$ (van Breugel \& Dey 1993) with 5" resolution, as extracted from the VLA archive (NED, Leahy \& Williams 1984), overlaid on a slightly smoothed $\mathrm{H} \alpha$ image from HST in the $F 702 W$ filter (data from the HST archive, PI: Crane). The observed positions are shown by the CO(1-0) 24" and $\mathrm{CO}(2-1) 12^{\prime \prime}$ IRAM $30 \mathrm{~m}$ beams (circles). Details of 3C 285/09.6 are shown in the circle on the left; they show that the spot is resolved into two or maybe three sub-structures.

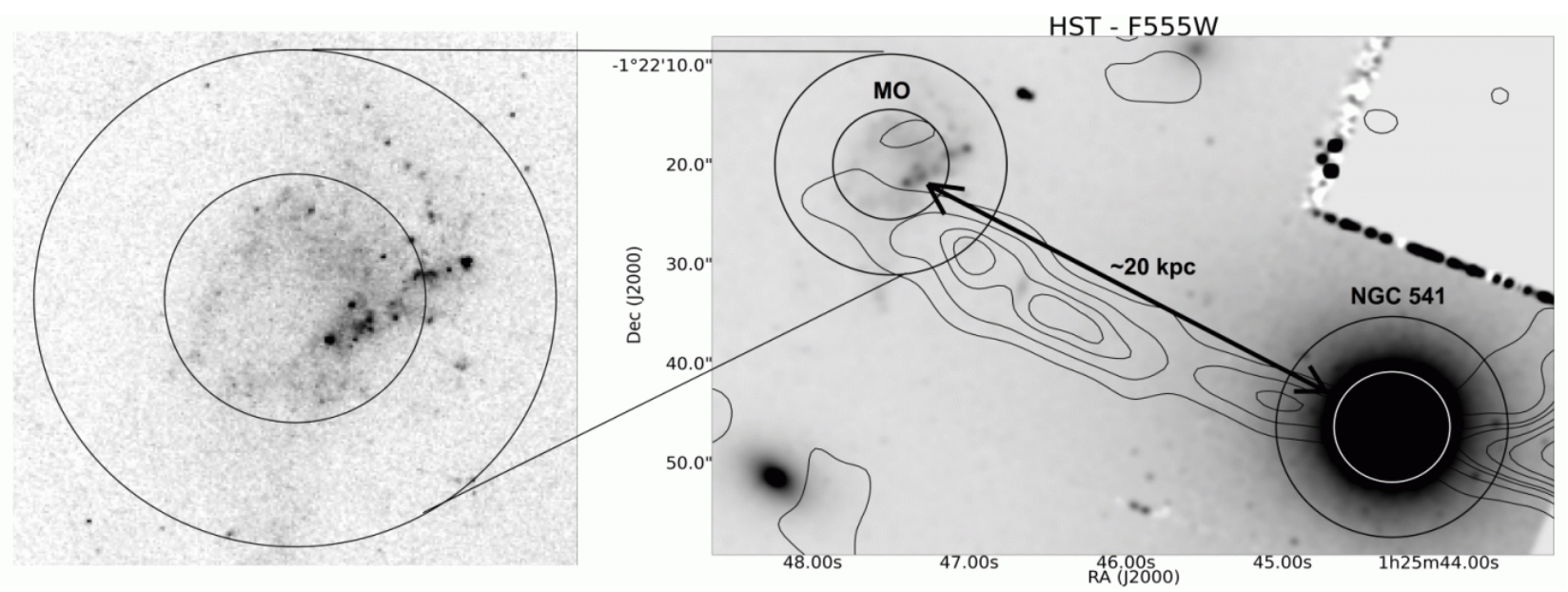

Fig. 2. Contour map of the eastern lobe of NGC 541 observed at $21 \mathrm{~cm}$ (van Breugel et al. 1985) with $3^{\prime \prime}$ resolution, as extracted from the VLA archive, overlaid on a slightly smoothed stellar continuum image from HST in the $F 555 \mathrm{~W}$ filter (data from the HST archive, PI: Baum). The observed positions are shown by the $\mathrm{CO}(1-0) 22^{\prime \prime}$ and $\mathrm{CO}(2-1) 11^{\prime \prime}$ IRAM 30 m beams (circles). Details of Minkowski's Object are shown on the left; they show that the spot is resolved into sub-structures.

Table 3. Journal of observations at IRAM $30 \mathrm{~m}$.

\begin{tabular}{lcccc}
\hline \hline Source & Line & Frequency & $\delta v$ & $\mathrm{rms}$ \\
\hline \multirow{2}{*}{ 3C 285 } & $\mathrm{CO}(1-0)$ & $106.780 \mathrm{GHz}$ & $44.9 \mathrm{~km} \mathrm{~s}^{-1}$ & $0.67 \mathrm{mK}$ \\
& $\mathrm{CO}(2-1)$ & $213.580 \mathrm{GHz}$ & & $1.70 \mathrm{mK}$ \\
3C 285/09.6 & $\mathrm{CO}(1-0)$ & $106.780 \mathrm{GHz}$ & $44.9 \mathrm{~km} \mathrm{~s}^{-1}$ & $0.49 \mathrm{mK}$ \\
& $\mathrm{CO}(2-1)$ & $213.580 \mathrm{GHz}$ & & $1.06 \mathrm{mK}$ \\
3C 285-2 & $\mathrm{CO}(1-0)$ & $106.780 \mathrm{GHz}$ & $44.9 \mathrm{~km} \mathrm{~s}^{-1}$ & $0.78 \mathrm{mK}$ \\
& $\mathrm{CO}(2-1)$ & $213.580 \mathrm{GHz}$ & & $1.58 \mathrm{mK}$ \\
\hline \multirow{2}{*}{ NGC 541 } & $\mathrm{CO}(1-0)$ & $113.211 \mathrm{GHz}$ & $42.4 \mathrm{~km} \mathrm{~s}^{-1}$ & $0.55 \mathrm{mK}$ \\
& $\mathrm{CO}(2-1)$ & $226.417 \mathrm{GHz}$ & $26.5 \mathrm{~km} \mathrm{~s}^{-1}$ & $1.12 \mathrm{mK}$ \\
MO & $\mathrm{CO}(1-0)$ & $113.143 \mathrm{GHz}$ & $44.9 \mathrm{~km} \mathrm{~s}^{-1}$ & $1.01 \mathrm{mK}$ \\
& $\mathrm{CO}(2-1)$ & $226.286 \mathrm{GHz}$ & & $1.35 \mathrm{mK}$ \\
\hline
\end{tabular}

Notes. The rms were determined with both polarisations. They are given in main beam temperature.

The data were reduced using the IRAM package CLASS. After dropping bad spectra, a linear baseline was subtracted from the average spectrum; for detections, the baseline was subtracted at velocities outside the range of the emission line $(-500$ to $500 \mathrm{~km} \mathrm{~s}^{-1}$ ). Then, each spectrum was smoothed to a spectral resolution of $\sim 40-45 \mathrm{~km} \mathrm{~s}^{-1}$, except for the $\mathrm{CO}(2-1)$ spectrum of NGC 541, which has a resolution of $\sim 25 \mathrm{~km} \mathrm{~s}^{-1}$. The resulting spectra are plotted in Figs. 3 and 4.

\subsection{Herschel}

The area around 3C 285 has been mapped with Herschel (Pilbratt et al. 2010). The observations were made with the SPIRE instrument (Griffin et al. 2010) at wavelengths 250, 350, and $500 \mu \mathrm{m}$ (see Fig. 5). We used these data, which are available in the online archive (ObsID: 1342256880). The fluxes of the central galaxy were then extracted on the region of the IRAM $30 \mathrm{~m}$ beam (see Table 4) before computing the spectral energy distribution (SED). To decrease the number of degrees of liberty, the Spitzer $70 \mu \mathrm{m}$ flux (Dicken et al. 2010) and the IRAS 60 and $100 \mu \mathrm{m}$ fluxes were also used.

\section{Results}

\subsection{CO luminosities}

The central galaxy 3C 285 was detected in both $\mathrm{CO}(1-0)$ and $\mathrm{CO}(2-1)$. Each line was fitted by a Gaussian to determine its characteristics. Line fluxes were measured by numerically integrating over the channels in the line profile, and the line widths 

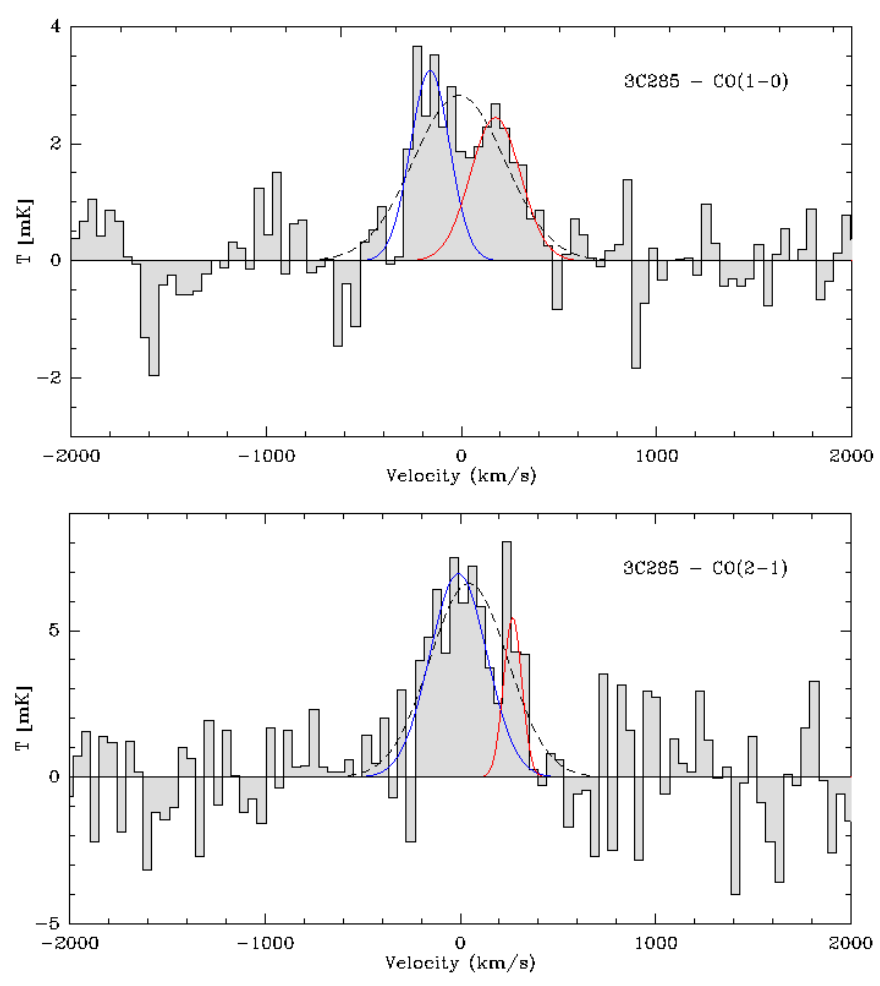

Fig. 3. Top: $\mathrm{CO}(1-0)$ spectrum for $3 \mathrm{C} 285$. The dashed line fits the total emission; the red and blue lines fit the double-horn profile. Bottom: $\mathrm{CO}(2-1)$ spectrum for $3 \mathrm{C} 285$. The spectra are tuned to the systemic redshift $(z=0.0794)$.

Table 4. Fluxes extracted from Herschel-SPIRE data. For 3C 285/09.6, an upper limit is given.

\begin{tabular}{lccc}
\hline \hline Source & $250 \mu \mathrm{m}$ & $350 \mu \mathrm{m}$ & $500 \mu \mathrm{m}$ \\
\hline 3C 285 & $1.00 \pm 0.013$ & $0.24 \pm 0.011$ & $0.14 \pm 0.011$ \\
3C 285/09.6 & $<3.79 \times 10^{-2}$ & $<3.41 \times 10^{-2}$ & $<3.43 \times 10^{-2}$ \\
\hline
\end{tabular}

Notes. Fluxes are in Jy.

were measured as full width at $50 \%$ of the peak flux. Then, $L_{\text {CO }}^{\prime}$ was calculated with the formula from Solomon et al. (1997). Results are summarised in Table 5 and give $L_{\mathrm{CO}}^{\prime}=(2.2 \pm 0.2) \times$ $10^{9} \mathrm{~K} \mathrm{~km} \mathrm{~s}^{-1} \mathrm{pc}^{2}$ for $3 \mathrm{C} 285$.

There is no detection for the other positions. Therefore, an upper limit of the line fluxes was calculated at $3 \sigma$ with the line width of the $\mathrm{H} \alpha$ emission equal to $64 \mathrm{~km} \mathrm{~s}^{-1}$ (van Breugel $\&$ Dey 1993). The computed upper limits are $L_{\mathrm{CO}}^{\prime} \leq 1.4 \times$ $10^{8} \mathrm{~K} \mathrm{~km} \mathrm{~s}^{-1} \mathrm{pc}^{2}$ and $L_{\mathrm{CO}}^{\prime} \leq 2.1 \times 10^{8} \mathrm{~K} \mathrm{~km} \mathrm{~s}^{-1} \mathrm{pc}^{2}$ for 3C 285/09.6 and 3C 285-2, respectively.

The molecular gas mass was estimated from the line luminosity $L_{\mathrm{CO}}^{\prime}$. A standard Milky Way conversion factor of 4.6 $M_{\odot}\left(\mathrm{K} \mathrm{km} \mathrm{s}^{-1} \mathrm{pc}^{2}\right)^{-1}$ (Solomon et al. 1997) was used to find a molecular gas mass of a few $10^{9} M_{\odot}$ for the central galaxy, while the other positions contain less than $10^{9} M_{\odot}$.

Evans et al. (2005) observed 3C 285 with the NRAO $12 \mathrm{~m}$ telescope. The $\mathrm{CO}(1-0)$ emission line was not detected in the central galaxy. Their non-detection is consistent with our results, when their beam dilution is taken into account (beam of $60^{\prime \prime}$ instead of our 24" beam), as well as their limited bandwidth of $1000 \mathrm{~km} \mathrm{~s}^{-1}$ for a line up to $1400 \mathrm{~km} \mathrm{~s}^{-1}$ at zero intensity.

NGC 541 was detected in $\mathrm{CO}(2-1)$ and partially detected in $\mathrm{CO}(1-0)$. The $\mathrm{CO}(2-1)$ luminosity of NGC 541
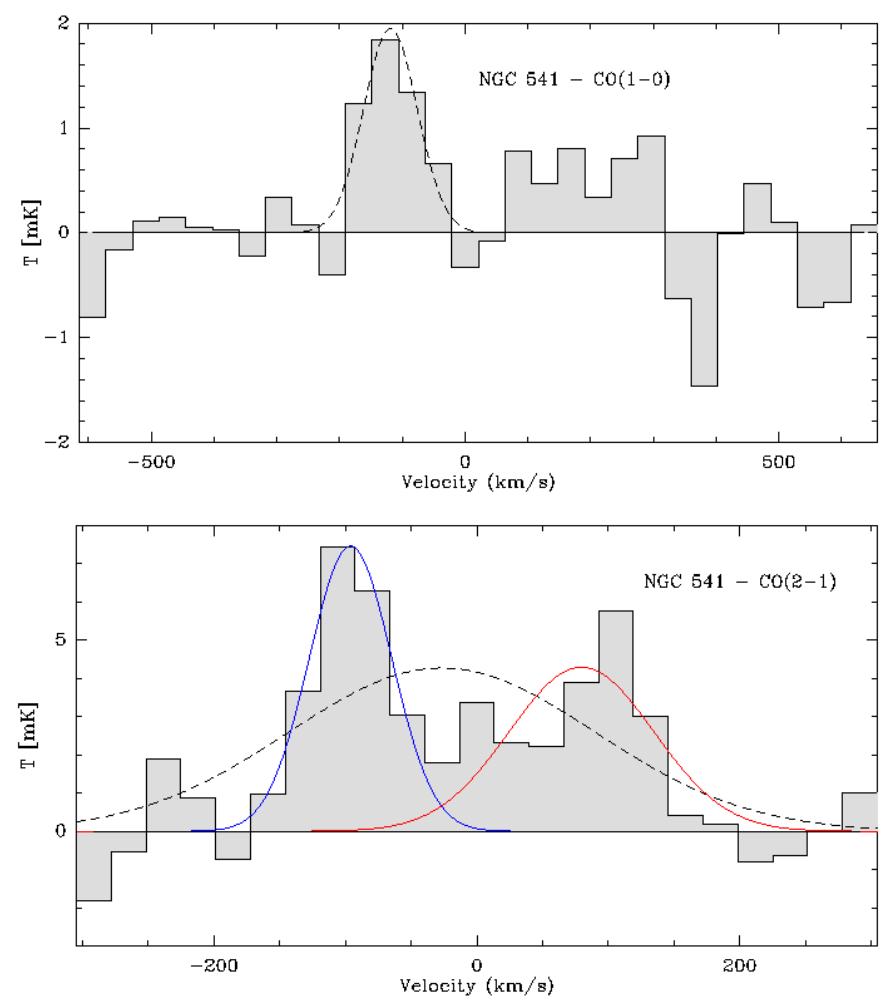

Fig. 4. Top: $\mathrm{CO}(1-0)$ spectrum of NGC 541 . The dashed line fits the total emission; the red and blue lines fit the double-horn profile. Bottom: $\mathrm{CO}(2-1)$ spectrum for NGC 541. The spectra are tuned to the systemic redshift $(z=0.0181)$.

is: $L_{\mathrm{CO}(2-1)}^{\prime}=(3.7 \pm 0.4) \times 10^{7} \mathrm{~K} \mathrm{~km} \mathrm{~s}^{-1} \mathrm{pc}^{2}$. Only the blueshifted part of the $\mathrm{CO}(1-0)$ spectrum was used to derive a lower limit of the luminosity: $L_{\mathrm{CO}(1-0)}^{\prime} \geq(1.4 \pm 0.3) \times$ $10^{7} \mathrm{~K} \mathrm{~km} \mathrm{~s}^{-1} \mathrm{pc}^{2}$. To calculate the mass of $\mathrm{H}_{2}$, we used the $\mathrm{CO}(2-1)$ luminosity assuming a ratio $\mathrm{CO}(2-1) / \mathrm{CO}(1-0)$ of 2.3 .

There is no detection for MO and an upper limit at $3 \sigma$ was calculated with a line width of $10 \mathrm{~km} \mathrm{~s}^{-1}$ estimated from the size and the stellar mass, taken as the dynamical mass. The computed upper limit is $L_{\mathrm{CO}}^{\prime} \leq 2.2 \times 10^{6} \mathrm{~K} \mathrm{~km} \mathrm{~s}^{-1} \mathrm{pc}^{2}$. Results are summarised in Table 5.

The molecular gas mass was estimated with a standard Milky Way conversion factor of $4.6 M_{\odot}\left(\mathrm{K} \mathrm{km} \mathrm{s}^{-1} \mathrm{pc}^{2}\right)^{-1}$ (Solomon et al. 1997). A molecular gas mass of a few $10^{8} M_{\odot}$ was found for NGC 541, while MO contains less than $10^{7} M_{\odot}$.

\subsection{Line intensities and line ratios}

Both CO lines are detected for 3C 285. The peak temperature and the integrated luminosity of the $\mathrm{CO}(2-1)$ line are about twice that for $\mathrm{CO}(1-0)$. If the lines were thermally excited, the brightness temperatures for a point source would be similar in the Rayleigh-Jeans regime. This means that the line main beam temperature ratio would be about 4 , as a result of the beam dilution. As the CO emission is not resolved (see Sect. 4.2), the factor of 2 indicates that the $J=2$ level is subthermally excited, as frequently observed in galaxies (Wiklind et al. 1995). But we note that the $\mathrm{CO}(2-1) / \mathrm{CO}(1-0)$ ratio is particularly low and might indicate a rather low density medium.

For NGC 541, a line ratio might be computed for the blueshifted part of the spectrum. The peak temperature in $\mathrm{CO}(2-1)$ is about four times greater and the integrated luminosity of the $\mathrm{CO}(2-1)$ line is about three times greater than that 
Q. Salomé et al.: Jet-induced star formation
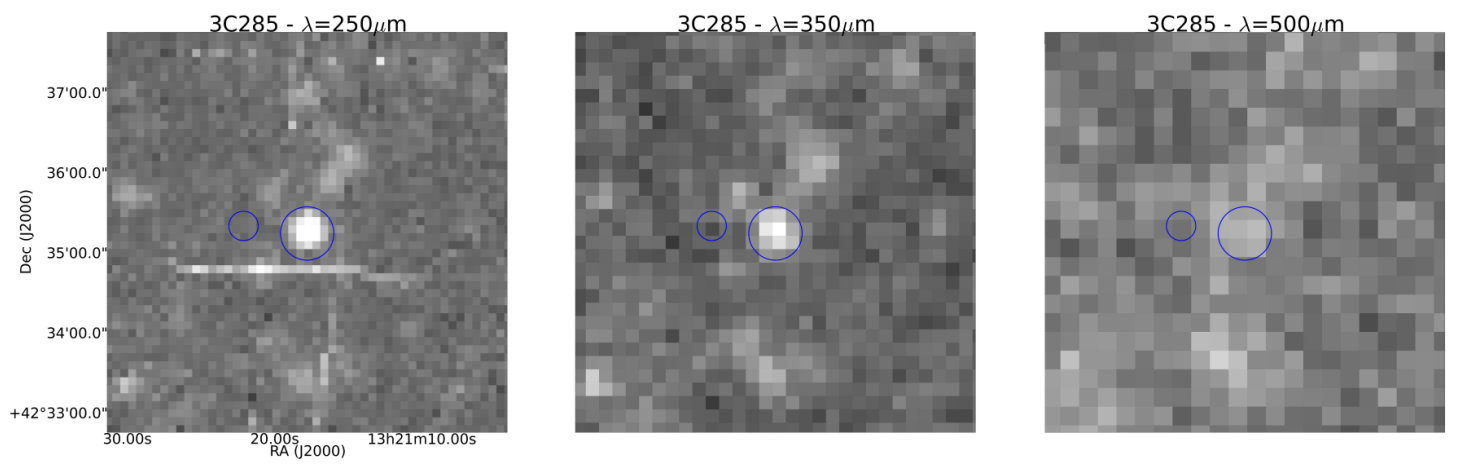

Fig. 5. Image of the Herschel-SPIRE data of the 3C 285 region at 250, 350, and $500 \mu \mathrm{m}$. The central galaxy and 3C 285/09.6 are shown with blue circles.

Table 5. Results of the observations at IRAM $30 \mathrm{~m}$.

\begin{tabular}{lccccccc}
\hline \hline Source & Line & $\begin{array}{c}T_{\mathrm{mb}} \\
(\mathrm{mK})\end{array}$ & $\begin{array}{c}\Delta v \\
\left(\mathrm{~km} \mathrm{~s}^{-1}\right)\end{array}$ & $\begin{array}{c}v_{\text {peak }} \\
\left(\mathrm{km} \mathrm{s}^{-1}\right)\end{array}$ & $\begin{array}{c}I_{\mathrm{CO}} \\
\left(\mathrm{K} \mathrm{km} \mathrm{s}^{-1}\right)\end{array}$ & $\begin{array}{c}L_{\mathrm{CO}}^{\prime} \\
\left(10^{8} \mathrm{~K} \mathrm{~km} \mathrm{~s}^{-1} \mathrm{pc}^{2}\right)\end{array}$ & $\begin{array}{c}M_{\mathrm{H}_{2}} \\
\left(10^{8} M_{\odot}\right)\end{array}$ \\
\hline 3C 285 & $\mathrm{CO}(1-0)$ & 2.82 & $553 \pm 51$ & $-11.6 \pm 26.4$ & $1.66 \pm 0.15$ & $22 \pm 2$ & $103 \pm 9$ \\
& $\mathrm{CO}(2-1)$ & 6.60 & $472 \pm 55$ & $44.2 \pm 25.5$ & $3.31 \pm 0.36$ & - & - \\
\hline 3C 285/09.6 & $\mathrm{CO}(1-0)$ & $<1.47$ & - & - & $<0.100$ & $<1.4$ & $<6.2$ \\
& $\mathrm{CO}(2-1)$ & $<3.18$ & - & - & $<0.216$ & - & - \\
\hline 3C 285-2 & $\mathrm{CO}(1-0)$ & $<2.34$ & - & - & $<0.159$ & $<2.1$ & $<9.9$ \\
& $\mathrm{CO}(2-1)$ & $<4.74$ & - & - & $<0.322$ & - & - \\
\hline NGC 541 & $\mathrm{CO}(1-0)$ & 1.95 & $101 \pm 25$ & $-118.8 \pm 12.8$ & $0.21 \pm 0.05$ & $0.14 \pm 0.03$ & $0.6 \pm 0.1$ \\
& $\mathrm{CO}(2-1)$ & 4.27 & $275 \pm 27$ & $-26.5 \pm 13.1$ & $1.25 \pm 0.12$ & $0.37 \pm 0.04$ & $1.7 \pm 0.2$ \\
\hline MO & $\mathrm{CO}(1-0)$ & $<3.03$ & - & - & $<0.032$ & $<0.02$ & $<0.10$ \\
& $\mathrm{CO}(2-1)$ & $<4.05$ & - & - & $<0.043$ & - & - \\
\hline
\end{tabular}

Notes. For non-detections, an upper limits is computed at $3 \sigma$, with a line width of $\Delta v=64 \mathrm{~km} \mathrm{~s}^{-1}$ for $3 \mathrm{C} 285 / 09.6$ and $3 \mathrm{C} 295-2$ (van Breugel \& Dey 1993), and a line width $\Delta v=10 \mathrm{~km} \mathrm{~s}^{-1}$ for MO.

for $\mathrm{CO}(1-0)$. In contrast to $3 \mathrm{C} 285$, the gas appears to be thermalised in NGC 541.

\subsection{Line width and morphology}

A broad line profile covers negative and positive velocities for 3C 285 and NGC 541. This could result from the rotation of the galaxies around their main axes, as suggested by the apparent double-horn profile. Using CLASS, both lines profiles were fitted with Gaussians (Figs. 3 and 4).

The blueshifted line peak temperature is slightly stronger for both frequencies. The double-horn profile of $\mathrm{CO}(1-0)$ emission is well defined for $3 \mathrm{C} 285$, but is less obvious in $\mathrm{CO}(2-1)$, probably because of the different beam sizes, or more likely because we work in a very low signal-to-noise regime. As discussed in Sect. 4.2, the CO emission is probably extended on scales $\sim 3.5 \mathrm{kpc}\left(\sim 2.3^{\prime \prime}\right)$ in radius. This means that the smaller $\mathrm{CO}(2-1)$ beam might have missed part of the rotating material.

Furthermore, the spectra show no kinematic effect of a molecular outflow at the level of the $30 \mathrm{~m}$ sensitivity.

\subsection{SED and IR luminosity}

We used the Herschel data to fit the SED of 3C 285 and determine its IR luminosity. The IR and radio emissions of the galaxy contain two parts: thermal emission from dust and synchrotron emission due to the AGN. The synchrotron contribution was first fitted by a power law with an index about -0.8 in frequency with data from the literature (Laing \& Peacock 1980; Hales et al. 1988; Gregory \& Condon 1991; Cohen et al. 2007). Then, the thermal part of the SED was computed with a fixed $\beta=1.5$ (cf. Fig. 6). It is characterised by a dust temperature $T_{\text {dust }} \sim 23 \mathrm{~K}$. This temperature gives a dust mass $M_{\text {dust }} \sim 2.05 \times 10^{8} M_{\odot}$ (Evans et al. 2005), which leads to a rather low gas-to-dust ratio $M_{\mathrm{H}_{2}} / M_{\text {dust }} \sim 50$. Wiklind et al. (1995) found that most of the elliptical galaxies have a gas-to-dust ratio of $\sim 700$. However, they also found some ellipticals with a ratio of $\sim 50$. They explained this difference by a lower dust temperature $(<30 \mathrm{~K})$ and by the fact that part of the FIR luminosity would come from grains associated with diffuse atomic gas (Wiklind et al. 1995).

The total infrared (TIR) luminosity was estimated by integrating the thermal part over the frequencies between 3 and $1100 \mu \mathrm{m}$ (as defined by Kennicutt \& Evans 2012): $L_{\mathrm{TIR}} \approx 1.32 \times$ $10^{11} L_{\odot}$ for $3 \mathrm{C} 285$. This is consistent with the FIR luminosity estimated by Sanders \& Mirabel (1996): $L_{\mathrm{FIR}}=9.51 \times 10^{10} L_{\odot}$ and Satyapal et al. (2005): $L_{\mathrm{FIR}}=7.69 \times 10^{10} L_{\odot}$.

It appears from the $L_{\mathrm{FIR}}$ vs. $L_{\mathrm{CO}}$ diagram (Solomon et al. 1997; Daddi et al. 2010) that 3C 285 is a normal and weakly interacting galaxy.

\subsection{Star formation rate}

The $\mathrm{H} \alpha$ emission emerging from gas photoionised by young and massive stars is often used as a tracer of star formation. The H $\alpha$ luminosity can thus be interpreted as a measure of the star formation rate with $S F R=L_{\mathrm{H} \alpha} / 1.86 \times 10^{41} \mathrm{erg} \mathrm{s}^{-1}$ (see Kennicutt \& Evans 2012, for a review).

The SFR may also be deduced from the TIR luminosity from dust emission. The emission from young stellar population is partly absorbed by dust which heats and emits in TIR via thermal 


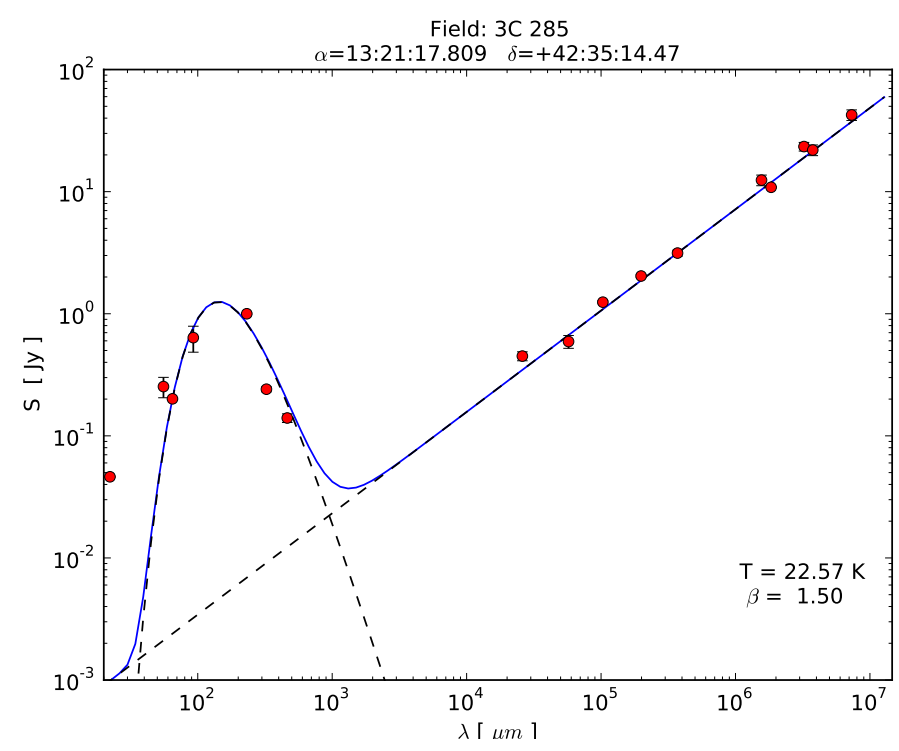

Fig. 6. Spectral energy distribution of the central galaxy $3 \mathrm{C} 285$. The dashed lines represent the synchrotron (power law) and thermal emission (modified black body). The Spitzer $24 \mu \mathrm{m}$ flux is plotted (not taken into account for fitting), indicating that there might be a warm dust component (Dicken et al. 2009).

Table 6. SFR in $M_{\odot} \mathrm{yr}^{-1}$ for the different objects observed with the IRAM $30 \mathrm{~m}$.

\begin{tabular}{lcccc}
\hline \hline Source & 3C 285 & 3C 285/09.6 & NGC 541 & MO \\
\hline$S F R_{\mathrm{H} \alpha}$ & 1.34 & 0.15 & $7 \times 10^{-3}$ & 0.35 \\
$S F R_{\mathrm{TIR}}$ & 19.7 & $<0.79$ & - & - \\
$S F R_{\mathrm{FIR}}$ & - & - & - & 0.09 \\
$S F R_{24 \mu \mathrm{m}}$ & 12.0 & 0.61 & 0.14 & 0.13 \\
\hline
\end{tabular}

Notes. The $\mathrm{H} \alpha$, TIR, FIR, and $24 \mu \mathrm{m}$ SFR were calculated following Kennicutt \& Evans (2012), Kennicutt (1998), and Calzetti et al. (2007). The total SFR is a combination of these SFR.

emission. The relationship between the TIR luminosity and the SFR is given by $S F R=L_{\mathrm{TIR}} / 6.7 \times 10^{9} L_{\odot}($ Kennicutt \& Evans 2012).

For the IR emission, the SFR can also be derived from the FIR or the $24 \mu \mathrm{m}$ emission: $S F R=L_{\mathrm{FIR}} / 5.8 \times 10^{9} L_{\odot}$ (Kennicutt 1998) and $S F R=1.27 \times 10^{-37}\left[L_{24} \mu \mathrm{m}\left(\mathrm{erg} \mathrm{s}^{-1}\right)\right]^{0.8850}$ (Calzetti et al. 2007). The $24 \mu \mathrm{m}$ luminosity was calculated with the WISE (Wright et al. 2010) data from the online archive. Table 6 summarises the different single-wavelength SFR.

SFR estimates differ by a factor of up to 20 , depending on the tracer used. This may be explained by dust absorption that obscures the $\mathrm{H} \alpha$ emission, or it might be that the IR emission is contaminated by the contribution from old stars.

To solve this problem, we used a multiwavelength estimation of the SFR. We corrected the $\mathrm{H} \alpha$ emission with the $24 \mu \mathrm{m}$ emission using a formula from Kennicutt \& Evans (2012): $L_{\mathrm{H} \alpha}^{\text {corr }}=$ $L_{\mathrm{H} \alpha}^{\mathrm{obs}}+0.020 L_{24} \mu \mathrm{m}$. The SFR was then calculated from the corrected $\mathrm{H} \alpha$ emission using the conversion formula above. Table 7 summarises the dust-attenuation correction of $\mathrm{H} \alpha$ and the corresponding SFR.

The depletion time is the time in which all the gas would be consumed if the SFR remains constant: $t_{\text {dep }} \sim M_{\text {gas }} / S F R$. For $3 \mathrm{C} 285$, the $\mathrm{CO}(1-0)$ emission line gives a gas mass $M_{\mathrm{H}_{2}}=$ $(10.3 \pm 0.9) \times 10^{9} M_{\odot}$ with the standard conversion factor. Therefore the depletion time is $(7.09 \pm 0.62) \times 10^{8} \mathrm{yr}$. As the
Table 7. Dust-attenuation corrections of the $\mathrm{H} \alpha$ emission using the formula from Kennicutt \& Evans (2012) for the different objects observed with the IRAM $30 \mathrm{~m}$.

\begin{tabular}{lcccc}
\hline \hline Source & 3C 285 & 3C 285/09.6 & NGC 541 & MO \\
\hline$L_{\mathrm{H} \alpha}^{\mathrm{obs}}\left(10^{40} \mathrm{erg} \mathrm{s}^{-1}\right)$ & 25 & 2.8 & 0.13 & 6.6 \\
$L_{24} \mu \mathrm{m}\left(10^{42} \mathrm{erg} \mathrm{s}^{-1}\right)$ & 110 & 3.8 & 0.74 & 0.66 \\
$L_{\mathrm{H} \alpha}^{\text {corr }}\left(10^{40} \mathrm{erg} \mathrm{s}^{-1}\right)$ & 245 & 10.4 & 1.61 & 7.92 \\
\hline$S F R\left(M_{\odot} \mathrm{yr}^{-1}\right)$ & 14.53 & 0.62 & 0.095 & 0.47 \\
$t_{\text {dep }}(\mathrm{Gyr})$ & 0.71 & $<1.0$ & 1.79 & 0.02 \\
\hline
\end{tabular}

Notes. The SFR is calculated with the conversion formula (Kennicutt \& Evans 2012).

Table 8. Molecular gas, stellar masses, and gas fraction for the different objects observed with the IRAM $30 \mathrm{~m}$.

\begin{tabular}{lcccc}
\hline \hline & NGC 541 & 3C 285 & 3C 285/09.6 & MO \\
\hline$M_{\text {gas }}\left(10^{8} M_{\odot}\right)$ & 1.7 & 103 & $<6.2$ & $<0.1$ \\
$M_{*}\left(10^{9} M_{\odot}\right)$ & 470 & 420 & 1.9 & 0.019 \\
$f_{\text {gas }}$ & 0.0004 & 0.025 & $<0.326$ & $<0.53$ \\
$S F R\left(M_{\odot} \mathrm{yr}^{-1}\right)$ & 0.095 & 14.53 & 0.62 & 0.47 \\
$t_{\text {dep }}(G y r)$ & 1.79 & 0.71 & $<1.0$ & $<0.02$ \\
$S S F R\left(10^{-2} \mathrm{Gyr}^{-1}\right)$ & 0.02 & 3.5 & 33 & $\sim 2500$ \\
\hline
\end{tabular}

Notes. The stellar masses are calculated with the mass-to-light ratio (Bell \& de Jong 2001). The depletion time is computed from the COderived gas masses and the SFR.

gas mass of $3 \mathrm{C} 285 / 09.6$ is $<6.2 \times 10^{8} M_{\odot}$, the depletion time is $<1.0 \times 10^{9} \mathrm{yr}$.

For NGC 541, the gas mass is given by $\mathrm{CO}(2-1)$ emission $M_{\mathrm{H}_{2}}=(1.7 \pm 0.2) \times 10^{8} M_{\odot}$, therefore the depletion time is $(1.79 \pm 0.21) \times 10^{9} \mathrm{yr}$. The mass of MO is $<1.0 \times 10^{7} \mathrm{M}_{\odot}$, consequently, the depletion time is $<2.1 \times 10^{7}$ yr. Table 8 summarises these results.

\section{Discussion}

\subsection{A Kennicutt-Schmidt law?}

The gas mass determined from the $\mathrm{CO}$ data may now be used to estimate the $M_{\mathrm{H}_{2}} / M_{*}$ ratio in each object. Croft et al. (2006) reported a stellar mass of $1.9 \times 10^{7} M_{\odot}$ for MO and Tadhunter et al. (2011) $M_{*} \sim 4.2 \times 10^{11} M_{\odot}$ for 3C 285 . For NGC 541 and $3 \mathrm{C} 285 / 09.6$, the stellar masses were calculated with the optical magnitudes and the mass-to-light ratios given by Bell \& de Jong (2001). Those masses are summarised in Table 8.

3C 285 has a molecular gas-to-stellar mass ratio of a few percent, while NGC 541 is very poor in gas. For MO and 3C 285/09.6, we only have upper limits, as shown in Table 8.

We conclude that 3C 285 lies on the main sequence of galaxies, while NGC 541 is a red sequence galaxy. This could indicate that a minor merger occured with 3C 285 and not with NGC 541. $3 \mathrm{C} 285 / 09.6$ lies on or above the main sequence. Finally, MO is a peculiar object, lying above the main sequence.

Note that we did not take into account the HI mass since no detections or upper limits in HI have been published yet, except for $\mathrm{MO}$, which has large amounts of atomic gas: $M_{\mathrm{HI}}=4.9 \times$ $10^{8} M_{\odot}$ (Croft et al. 2006).

We calculated the gas and SFR surface densities $\left(\Sigma_{\text {gas }}, \Sigma_{\mathrm{SFR}}\right)$. For $3 \mathrm{C} 285$, both quantities were smoothed over the $\mathrm{CO}(1-0)$ IRAM $30 \mathrm{~m}$ beam, which gives $\Sigma_{\mathrm{H}_{2}} \approx 58.0 \pm 5.1 M_{\odot} \mathrm{pc}^{-2}$ 


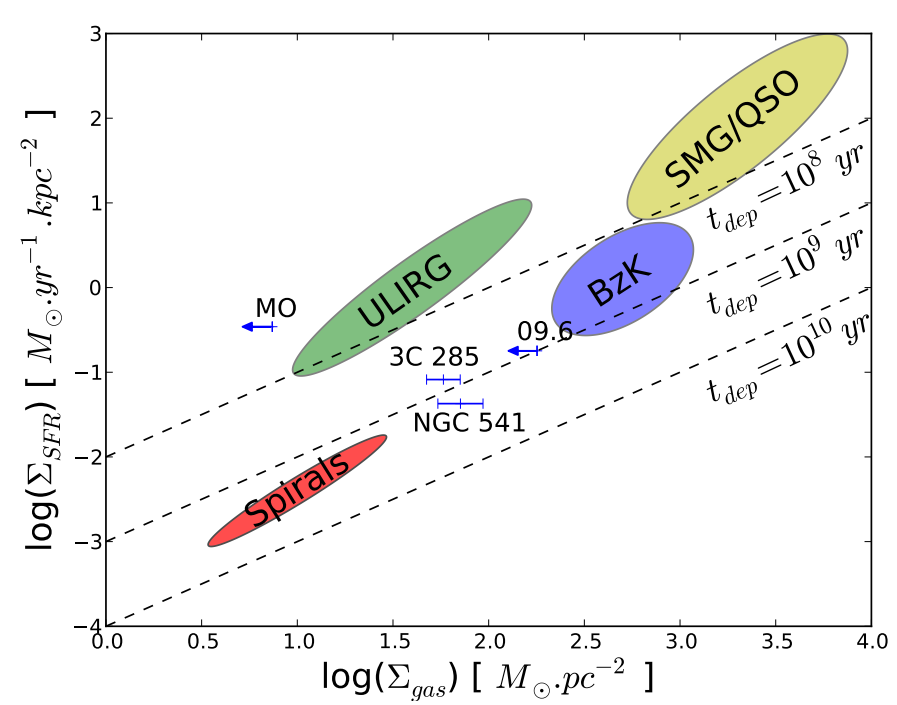

Fig. 7. $\Sigma_{\text {SFR }}$ vs. $\Sigma_{\text {gas }}$ for the four sources. The diagonal dashed lines show lines of constant SFE, indicating the level of $\Sigma_{\text {SFR }}$ needed to consume $1 \%, 10 \%$, and $100 \%$ of the gas reservoir in $10^{8}$ years. Thus, the lines also correspond to constant gas depletion times of, from top to bottom, $10^{8}, 10^{9}$, and $10^{10} \mathrm{yr}$. The coloured regions come from Daddi et al. (2010).

and $\Sigma_{\mathrm{SFR}} \approx 0.082 M_{\odot} \mathrm{yr}^{-1} \mathrm{kpc}^{-2}$. For $3 \mathrm{C} 285 / 09.6$, the surface densities were estimated on the area of the $\mathrm{H} \alpha$ emission (in a radius of $\sim 1.65^{\prime \prime}$ ), giving $\Sigma_{\mathrm{H}_{2}}<179 M_{\odot} \mathrm{pc}^{-2}$ and $\Sigma_{\mathrm{SFR}} \approx$ $0.179 M_{\odot} \mathrm{yr}^{-1} \mathrm{kpc}^{-2}$.

Plotting this in the $\Sigma_{\text {SFR }}$ vs. $\Sigma_{\text {gas }}$ diagram (see Fig. 7, Bigiel et al. 2008; Daddi et al. 2010), both positions follow a SchmidtKennicutt law $\Sigma_{\mathrm{SFR}} \propto \Sigma_{\mathrm{H}_{2}}^{N}$ (Kennicutt 1998). To accurately determine the SFE in 3C 285/09.6, high-resolution interferometric data are required.

In NGC 541, the gas and SFR surface densities were smoothed over the $\mathrm{CO}(2-1)$ IRAM $30 \mathrm{~m}$ beam, which gives $\Sigma_{\mathrm{H}_{2}} \approx 71.2 \pm 8.4 M_{\odot} \mathrm{pc}^{-2}$ and $\Sigma_{\mathrm{SFR}} \approx 4.26 \times$ $10^{-2} M_{\odot} \mathrm{yr}^{-1} \mathrm{kpc}^{-2}$. For MO, the surface densities were estimated on the area of the stellar emission (in a radius of $\sim 4.08^{\prime \prime}$ ), giving $\Sigma_{\mathrm{H}_{2}}<7.4 M_{\odot} \mathrm{pc}^{-2}$ and $\Sigma_{\mathrm{SFR}} \approx 0.345 M_{\odot} \mathrm{yr}^{-1} \mathrm{kpc}^{-2}$.

As a conclusion, Fig. 7 shows that the two star-forming regions 3C 285/09.6 and MO (lying along the AGN radio-jets of 3C 285 and NGC 541) must have depletion time scales of the order of or shorter than typical spiral galaxies. This supports the AGN positive feedback scenario that predicts an enhanced star formation activity along the shocked region inside the radio-jets. More sensitive observations to detect and map the $\mathrm{CO}$ emission are necessary, however, to accurately measure the effect of the jet on the gas, its impact on the cooling, and thus on the triggered SFE.

\subsection{A model for computing velocity spectra}

To interpret the kinematics of our CO data, we used a simple analytical model that computes the velocity spectrum from the rotation velocity profile of the galaxy (Wiklind et al. 1997). The rotation velocity profile was determined from the stellar mass distribution, assuming it follows a Plummer distribution.

The gas distribution was assumed to be axisymmetric of the surface density $n(r)$ in a disc with negligible thickness. For each velocity $\mathrm{d} v$, the code calculates the density contained in the isovelocity (Eq. (1)). The velocity spectrum corresponds to

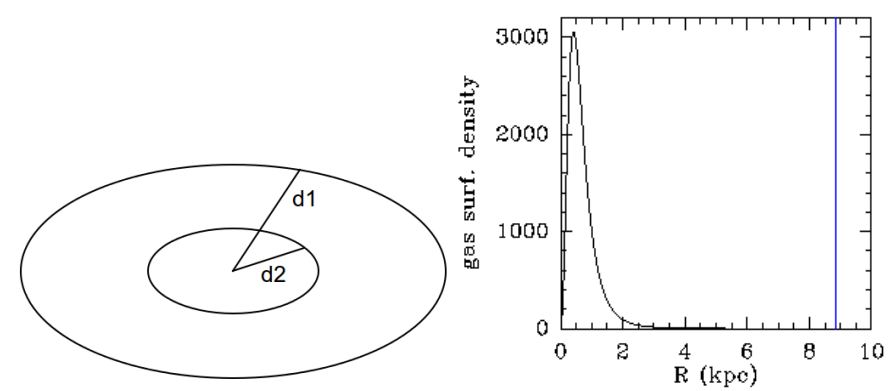

Fig. 8. Left: sketch of a ring with the characteristic distances $d_{1}$ and $d_{2}$. Right: surface density profile as derived by the model for a Toomre ring of mass $1.03 \times 10^{10} M_{\odot}$ with $d_{1}=0.9 \mathrm{kpc}$ and $d_{2}=0.5 \mathrm{kpc}$. The vertical blue line represents the size of the $\mathrm{CO}(2-1)$ beam for $3 \mathrm{C} 285$.

the histogram of the velocities. To take into account the gas dispersion, the computed spectrum was then convolved with a Gaussian of standard $\sigma=10 \mathrm{~km} \mathrm{~s}^{-1}$.

$\frac{\mathrm{d} N}{\mathrm{~d} v}(v)=\int \frac{n(r) r \mathrm{~d} r}{v_{\text {rot }}(r) \sin i\left(1-\frac{v}{v_{\text {rot }}(r) \sin i}\right)^{2}}$.

We wished to study the concentration of gas in the galaxy and determine whether the gas is distributed in a disc or a ring. The disc was modelled with a Toomre disc of order 2: $n(r)=$ $n_{0}\left(1+\frac{r^{2}}{d^{2}}\right)^{-5 / 2}$ (Toomre 1964). A ring is the difference between two Toomre discs (see sketch in Fig. 8).

We ran grids of this model and varied the distances $d_{1}$ and $d_{2}$. $d_{1}$ varied from a few hundred parsecs to $10 \mathrm{kpc}, d_{2}$ from 0 to a few tens of parsecs below $d_{1}\left(d_{2}=0\right.$ corresponds to a disc). Both $d_{1}$ and $d_{2}$ influence the morphology of gas. For low values of $d_{1}$, the gas is distributed in a narrow dense ring, for larger distances, the ring is broad with a broadness of a few kiloparsecs. In addition, for inner radii larger than $\sim 2 \mathrm{kpc}$, the gas ring extends far enough to be slightly resolved by the IRAM $30 \mathrm{~m}$ telescope.

The inclination angle will also influence the spectra characteristics. As the radial velocity is proportional to the sine of the angle, the peaks approach each other as the inclination decreases. The depth does not change significantly with inclination, except for very low angles, when the peaks start to overlap.

\subsection{A compact molecular ring in 3C 285 and NGC 541}

Roche $\&$ Eales (2000) used $V$ - and $R$-band data to investigate the radial profiles of radio galaxies. For $3 \mathrm{C} 285$, the best-fit model gives a half-light radius of $\sim 8.3 \mathrm{kpc}$, with a stellar mass of $\sim 4.2 \times 10^{11} M_{\odot}$. To compare the models with the data, we used the peak velocity and the relative well depth (see Fig. 10).

The observational spectrum presents peak velocities of $\sim 160-175 \mathrm{~km} \mathrm{~s}^{-1}$ and a relative well depth of $1 / 3$. The ranges of parameters that fit the observations are $d_{1}=0.7-1.0 \mathrm{kpc}$ and $d_{2}=0.5-0.7 \mathrm{kpc}$ for an inclination angle larger than $70^{\circ}$ (see left panel of Fig. 9), which is consistent with the optical image. Figure 10 represents the spectra for a ring of $1.03 \times 10^{10} M_{\odot}$ with $d_{1}=0.9 \mathrm{kpc}$ and $d_{2}=0.5 \mathrm{kpc}$. The density profile (Fig. 8) indicates that the gas is distributed in a narrow ring that extends at distances up to $\sim 2 \mathrm{kpc}$ with an average radius $\sim 0.7 \mathrm{kpc}$, but this needs to be confirmed by interferometric data.

For NGC 541, the half-light radius is $\sim 5-8 \mathrm{kpc}$ (Loubser $\&$ Sánchez-Blázquez 2012), with a stellar mass of $\sim 4.7 \times 10^{11} M_{\odot}$ (Bell \& de Jong 2001). 

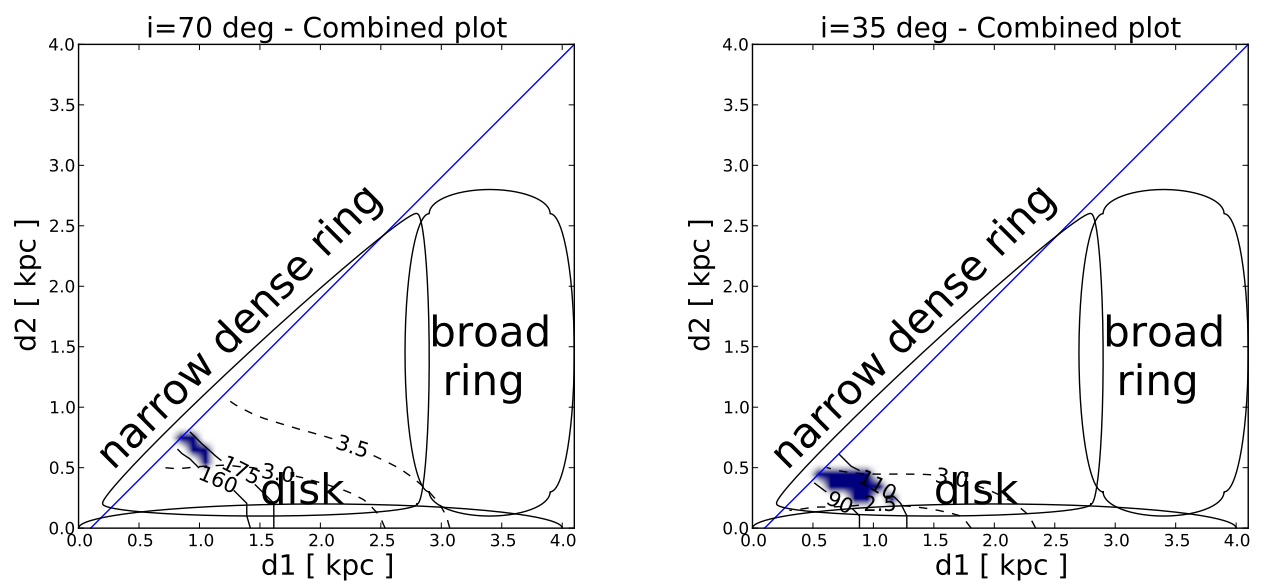

Fig. 9. Parameter space of the model grids for 3C 285 (left) and NGC 541 (right). The axes indicate the value of the outer $d_{1}$ and inner $d_{2}$ distances of the Toomre ring (see sketch in Fig. 8). Since $d_{1}>d_{2}$, the explored parameters lie below the blue line (bottom-right part of the plot). The black solid lines show solutions for a peak velocity of 160 and $175 \mathrm{~km} \mathrm{~s}^{-1}$ (left), and 90 and $110 \mathrm{~km} \mathrm{~s}^{-1}$ (right). The black dashed lines show the solutions for a well depth (see Fig. 10) of 30\% and 35\% of the peak (left), and $25 \%$ and $30 \%$ of the peak (right). These values correspond to the observational constraints extracted from the $30 \mathrm{~m}$ telescope spectra. The dark blue surface is the intersection that fits both solutions.
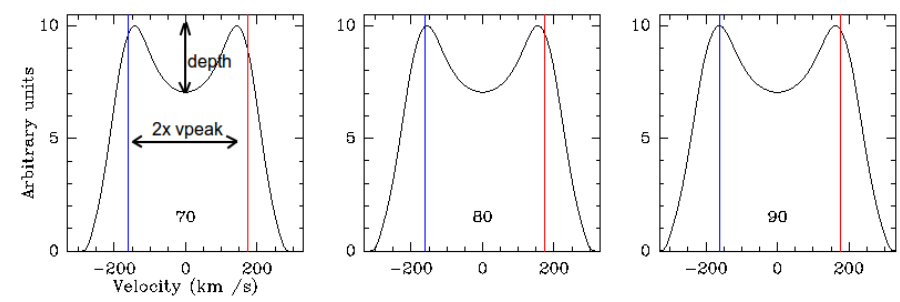

Fig. 10. Velocity spectra computed from the model for inclination angles of $70^{\circ}, 80^{\circ}$ and $90^{\circ}$. The vertical blue and red lines indicate the position of the double-horned profile peaks in the data.

The observational spectrum presents a peak velocity of $\sim 100 \mathrm{~km} \mathrm{~s}^{-1}$ and a relative well depth of $1 / 4-1 / 3$. The range of parameters that fit the observations are $d_{1}=0.4-1.1 \mathrm{kpc}$ and $d_{2}=0.2-0.4 \mathrm{kpc}$ for an inclination angle between $30^{\circ}$ and $40^{\circ}$ (see right panel of Fig. 9), which is consistent with the optical image. The gas is distributed in a narrow ring that extends at distances up to $\sim 2 \mathrm{kpc}$ with an average radius $\sim 0.5 \mathrm{kpc}$, but this also needs to be confirmed by interferometric data.

\section{Conclusions}

We used the IRAM 30 m telescope to observe the centre region of two radio-galaxies: 3C 285 and NGC 541. We also pointed towards two star-forming regions standing along the radio-jet direction of each of these objects: 3C 285/09.6 at a distance of $\sim 70 \mathrm{kpc}$ from 3C 285, and Minkowski's Object (MO) at $20 \mathrm{kpc}$ from NGC 541.

NGC 541 was detected in $\mathrm{CO}(2-1)$. The $\mathrm{CO}(1-0)$ emission line is marginally visible when our observations are combined with the non-detection from Ocaña Flaquer et al. (2010) on the same object.

We derived a total molecular gas mass of $\sim 10^{8} M_{\odot}$, leading to a gas fraction smaller than $1 \%$. With a very low star formation rate, this object thus appears to be a typical red and dead galaxy. However, its depletion time scale ( $2 \mathrm{Gyr})$ is typical of normal star-forming galaxies. Therefore the very low star formation rate is mostly due to the small gas fraction in this object. The $\mathrm{CO}$ line profile has a typical double-horn shape that indicates a possible rotating disk or ring. To reproduce the molecular gas velocity profile, we ran simplified analytical models, constrained by estimates of the stellar mass of the system, its effective radius, and its inclination. These models reproduced the $\mathrm{CO}$ line profiles with a rather compact ring-like distribution $(\sim 1-2 \mathrm{kpc})$.

The origin of this gas is not discussed here, but as already deduced for other objects such as Centaurus A, rotating rings of molecular gas are the expected remnants of a recent minor merger activity.

3C 285 was detected in $\mathrm{CO}(1-0)$ and $\mathrm{CO}(2-1)$ with a total molecular gas mass of $\sim 10^{10} M_{\odot}$, meaning a gas fraction of $\sim 2.5 \%$. Surprisingly for this type of source, 3C 285 has a fairly high SFR of $\sim 15 M_{\odot} \mathrm{yr}^{-1}$. With a depletion time of less than one Gyr, this source appears to be a typical star-forming galaxy, as shown by its position in a KS-diagram. The simple analytical models that reproduce the $\mathrm{CO}$ line profiles are also the ones where the gas is located in a compact molecular ring/disk of $1-2 \mathrm{kpc}$. This means that star formation may proceed in this very compact region hidden inside a larger dust-lane seen in the optical image that crosses the entire galaxy. Note that 3C 285 has a much more massive molecular gas reservoir than NGC 541, standing in a disk of about the same size. The molecular gas density must therefore be higher, which could explain why the $\operatorname{SFE}\left(1 / t_{\text {dep }}\right)$ is higher in this object.

MO and 3C 285/09.6 have not been detected in CO by the $30 \mathrm{~m}$ telescope. However, we reached interesting rms for these two sources, leading to upper limits of molecular gas amount of $\sim 10^{7}-10^{8} M_{\odot}$. This means that 3C 285/09.6 and MO have a depletion time of $\leq 1 \mathrm{Gyr}$ and $\leq 0.02$ Gyr. In a KS-diagram, $3 \mathrm{C} 285 / 09.6$ lies among or above the normal star-forming galaxies, MO among the highly efficient star-forming objects. This result shows that the star formation observed in the radio-lobes of 3C 285 and NGC 541 is at least as efficient as inside spiral galaxies and even boosted in the case of MO.

If molecular gas is present, as suggested by the star formation activity, its origin in 3C 285/09.6 and MO is still an open question. However, the differences in the molecular-toatomic gas fraction, the gas-to-dust ratio, or the specific star formation rate (sSFR) in these two objects indicate different scenarios. 3C 285/09.6 could be the remnant of a small galaxy that has lost most of its gas in a tidal interaction and is being 
compressed by the interaction with the 3C 285 radio-lobe. In the case of MO, the atomic gas, the short depletion time scale, and the very high sSFR may indicate a recent star-forming event that has not produced many stars yet. While 3C 285/09.6 has a stellar mass of $\sim 10^{9} M_{\odot}$, typical of a small galaxy, that of MO is 100 times smaller with a stellar mass of only $\sim 10^{7} M_{\odot}$. The small amounts of molecular gas in MO could be explained if the gas were mainly atomic or if the metallicity were too low to maintain the standard conversion factor. In this case, MO could have condensed, after the interaction with the radio-lobes, from the low-metallicity intergalactic medium surrounding NGC 541, as already suggested for the filaments of several brightest cluster galaxies.

This is consistent with the modelling reported by Fragile et al. (2004), where the authors applied their hydrodynamic simulations of radiative shock-cloud interactions to $\mathrm{MO}$ as a test case. They concluded that MO could result from an interaction of $\mathrm{a} \sim 10^{5} \mathrm{~km} \mathrm{~s}^{-1}$ jet with an ensemble of moderately dense $\left(10 \mathrm{~cm}^{-3}\right)$ and warm $\left(10^{4} \mathrm{~K}\right)$ intergalactic clouds, the large HI mass in MO being explained by the radio-jet triggered radiative cooling of the warm surrounding gas.

Acknowledgements. We thank the anonymous referee for his/her useful remarks. We also thank Santiago Garcia-Burillo for helpful comments. Herschel is an ESA space observatory with science instruments provided by Europeanled Principal Investigator consortia and with important participation from NASA. This research has made use of the NASA/IPAC Extragalactic Database (NED) which is operated by the Jet Propulsion Laboratory, California Institute of Technology, under contract with the National Aeronautics and Space Administration. This research has made use of the NASA/IPAC Infrared Science Archive, which is operated by the Jet Propulsion Laboratory, California Institute of Technology, under contract with the National Aeronautics and Space Administration. This publication makes use of data products from the Widefield Infrared Survey Explorer, which is a joint project of the University of California, Los Angeles, and the Jet Propulsion Laboratory/California Institute of Technology, funded by the National Aeronautics and Space Administration. F.C. acknowledges the European Research Council for the Advanced Grant Program Number 267399-Momentum.

\section{References}

Baum, S. A., \& Heckman, T. 1989, ApJ, 336, 681

Bell, E. F., \& de Jong, R. S. 2001, ApJ, 550, 212

Best, P. N., \& Heckman, T. M. 2012, MNRAS, 421, 1569

Bicknell, G. V., Sutherland, R. S., van Breugel, W. J. M., et al. 2000, ApJ, 540, 678

Bigiel, F., Leroy, A., Walter, F., et al. 2008, AJ, 136, 2846

Calzetti, D., Kennicutt, R. C., Engelbracht, C. W., et al. 2007, ApJ, 666, 870

Capetti, A., Kleijn, G. V., \& Chiaberge, M. 2005, A\&A, 439, 935
Charmandaris, V., Combes, F., \& van der Hulst, J. M. 2000, A\&A, 356, L1

Cohen, A. S., Lane, W. M., Cotton, W. D., et al. 2007, AJ, 134, 1245

Croft, S., van Breugel, W., de Vries, W., et al. 2006, ApJ, 647, 1040

Daddi, E., Elbaz, D., Walter, F., et al. 2010, ApJ, 714, L118

De Breuck, C., Downes, D., Neri, R., et al. 2005, A\&A, 430, L1

Dicken, D., Tadhunter, C., Axon, D., et al. 2009, ApJ, 694, 268

Dicken, D., Tadhunter, C., Axon, D., et al. 2010, ApJ, 722, 1333

Emonts, B. H. C., Norris, R. P., Feain, I., et al. 2014, MNRAS, 438, 2898

Engelbracht, C., Rieke, G., Gordon, K., et al. 2008, ApJ, 678, 804

Evans, A. S., Mazzarella, J. M., Surace, J. A., et al. 2005, ApJS, 159, 197

Fabian, A. 2012, ARA\&A, 50, 455

Fragile, P. C., Murray, S. D., Anninos, P., \& van Breugel, W. 2004, ApJ, 604, 74

Gaibler, V., Khochfar, S., Krause, M., \& Silk, J. 2012, MNRAS, 425, 438

Gregory, P. C., \& Condon, J. J. 1991, ApJS, 75, 1011

Griffin, M. J., Abergel, A., Abreu, A., et al. 2010, A\&A, 518, L3

Hales, S. E. G., Baldwin, J. E., \& Warner, P. J. 1988, MNRAS, 234, 919

Heckman, T. M., \& Best, P. N. 2014, ARA\&A, 52, 589

Ivison, R. J., Smail, I., Amblard, A., et al. 2012, MNRAS, 425, 1320

Kennicutt, Jr., R. C. 1998, ApJ, 498, 541

Kennicutt, R. C., \& Evans, N. J. 2012, ARA\&A, 50, 531

Klamer, I. J., Ekers, R. D., Sadler, E. M., \& Hunstead, R. W. 2004, ApJ, 612, L97

Laing, R. A., \& Peacock, J. A. 1980, MNRAS, 190, 903

Leahy, J. P., \& Williams, A. G. 1984, MNRAS, 210, 929

Loubser, S. I., \& Sánchez-Blázquez, P. 2012, MNRAS, 425, 841

McNamara, B. 2002, New Astron. Rev., 46, 141

McNamara, B. R., \& O'Connell, R. W. 1993, AJ, 105, 417

Mould, J. R., Ridgewell, A., Gallagher III, J. S., et al. 2000, ApJ, 536, 266

Ocaña Flaquer, B., Leon, S., Combes, F., \& Lim, J. 2010, A\&A, 518, A9

Papadopoulos, P. P., Greve, T. R., Ivison, R. J., \& De Breuck, C. 2005, A\&A, 444, 813

Pilbratt, G. L., Riedinger, J. R., Passvogel, T., et al. 2010, A\&A, 518, L1

Roche, N., \& Eales, S. A. 2000, MNRAS, 317, 120

Salomé, P., \& Combes, F. 2004, A\&A, 415, L1

Salomé, P., Revaz, Y., Combes, F., et al. 2008, A\&A, 483, 793

Salomé, P., Combes, F., Revaz, Y., et al. 2011, A\&A, 531, A85

Sanders, D. B., \& Mirabel, I. F. 1996, ARA\&A, 34, 749

Satyapal, S., Dudik, R. P., O’Halloran, B., \& Gliozzi, M. 2005, ApJ, 633, 86

Schiminovich, D., van Gorkom, J. H., van der Hulst, J. M., \& Kasow, S. 1994, ApJ, 423, L101

Simkin, S. M. 1976, ApJ, 204, 251

Solomon, P. M., Downes, D., Radford, S. J. E., \& Barrett, J. W. 1997, ApJ, 478, 144

Tadhunter, C., Holt, J., Delgado, R. G., et al. 2011, MNRAS, 412, 960

Toomre, A. 1964, ApJ, 139, 1217

van Breugel, W. J. M., \& Dey, A. 1993, ApJ, 414, 563

van Breugel, W., Filippenko, A. V., Heckman, T., \& Miley, G. 1985, ApJ, 293, 83

Véron-Cetty, M., \& Véron, P. 2010, A\&A, 518, A10

Wiklind, T., Combes, F., \& Henkel, C. 1995, A\&A, 297, 643

Wiklind, T., Combes, F., Henkel, C., \& Wyrowski, F. 1997, A\&A, 323, 727

Wright, E. L., Eisenhardt, P. R. M., Mainzer, A. K., et al. 2010, ApJ, 140 , 1868

Zinn, P., Middelberg, E., Norris, R. P., \& Dettmar, R. 2013, ApJ, 774, 66 